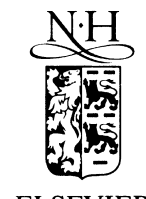

Nuclear Instruments and Methods in Physics Research B 153 (1999) 284-291

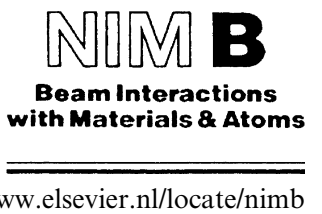

www.elsevier.nl/locate/nimb

\title{
Comparison of gold and carbon cluster impacts on graphite using Molecular Dynamics simulation
}

\author{
Roger Webb ${ }^{\text {a,*, }}$, Michelle Kerford ${ }^{\mathrm{a}}$, Allan Way ${ }^{\mathrm{a}}$, Ian Wilson ${ }^{\mathrm{b}}$ \\ ${ }^{a}$ Department of Electronic and Electrical Engineering, University of Surrey, Guildford GU2 5XH, UK \\ ${ }^{\mathrm{b}}$ Department of Electronic and Electrical Engineering, Chinese University of Hong Kong, New Territories, Hong Kong, \\ People's Republic of China
}

\begin{abstract}
SIMS experiments using cluster ions show promising results in de-sorbing intact large molecules from the surface of various materials. The optimum size, shape and energy of the clusters and the material surface has not been studied in any detail. Presented here are the results of a comparative study of gold and carbon clusters of similar total mass and energy, in particular $\mathrm{Au}_{4}$ and $\mathrm{C}_{60}$ clusters and $\mathrm{Au}_{5}$ and $\mathrm{C}_{100}$ clusters are compared and contrasted. The behaviour of the graphite lattice, used as the target substrate material, during the impacts is studied in detail and differences in depth of penetration, damage and energy propagation are reported. (C) 1999 Elsevier Science B.V. All rights reserved.
\end{abstract}

\section{Introduction}

There is a growing interest in the interaction of energetic clusters with solid surfaces due to the use of cluster beams in ion implantation [1], in ion beam analysis [2] and in deposition [3]. Consequently there has been a lot of work in this area in recent years, both experimentally [4] and computationally [5]. It well known that the interaction of heavy ions with dense or frozen gas targets can result in non-linear cascade formation, thermal spikes and large sputtering events [6]. Low energy cluster and molecular impacts on surfaces produce cascades from each component of the cluster, these

\footnotetext{
${ }^{*}$ Corresponding author. Tel.: +44-1483-259830; fax: +44-11483-34139; e-mail: r.webb@ee.surrey.ac.uk
}

will tend to overlap in space and time giving rise to non-linear effects in the majority of target materials. The effects of single particle impacts on surfaces are largely dominated by linear cascade theory [7]. Cluster impacts will, on the other hand, be dominated by non-linear effects. These effects will lessen as the energy increases and the overlap of the cascades caused by each constituent of the cluster diminishes. There have been a number of simulation studies looking at the ejection of organic materials from metal surfaces due to single particle [8] and small cluster [9] irradiation. There has been some work looking at the secondary ion emission from surfaces due to different size clusters [10]. To date, however, there has been no study of the effects of clusters with the same masses but different numbers of constituent particles.

The aim of this study is to look at the effects of such clusters using computer simulation tech- 
niques. Previous simulation studies have looked at the effects of bombardment of graphite targets with different mass fullerene molecules [11], in particular $\mathrm{C}_{60}$ projectiles have been used [12]. Here we will look at the effects of similar total mass clusters containing different mass constituents. We will compare $\mathrm{C}_{60}$ and $\mathrm{Au}_{4}$ and $\mathrm{C}_{100}$ and $\mathrm{Au}_{6}$ clusters impacting on a graphite surface. The details of the component masses and properties are given in Table 1, below. As can be seen, the mass of $\mathrm{Au}_{4}$ and $\mathrm{C}_{60}$ are within $10 \%$ of each other as are $\mathrm{C}_{100}$ and $\mathrm{Au}_{6}$. Energies of the projectiles are chosen so that the velocities of the clusters are similar but the energy per component atom is different. The effects of displacements and penetration depth are looked at in each case. We use Molecular Dynamics computer simulation to investigate the differences between clusters with the same initial momenta. The clusters contain different numbers of atoms and so will fragment into different numbers of cascades. The atoms in the clusters themselves have different atomic numbers and so will not interact with the target in the same way either.

\section{The simulation model}

The Molecular Dynamics simulation model used in this study has been described in detail in other publications $[13,14]$ and so only a brief description will be given here. The simulation solves the equations of motion simultaneously for each particle and stores information on position, velocity and energy states at different time steps. The program uses the Brenner many-body potentials [15] to model the $\mathrm{C}-\mathrm{C}$ and $\mathrm{C}-\mathrm{H}$ interactions. For the dynamic systems used here these potentials are known to give both stable graphite and diamond lattices as well as a good description of the multimer properties of carbon and the correct mechanical properties of graphite and diamond. The properties of the $\mathrm{Au}-\mathrm{Au}$ and the $\mathrm{Au}-\mathrm{C}$ interactions are given by pair-wise interaction potentials given by the ZBL universal function [16]. These interaction potentials do not describe the bonding nature of the $\mathrm{Au}-\mathrm{Au}$ and $\mathrm{Au}-\mathrm{C}$ particles very well. Hence they can not be used to describe the bonding configuration of the implanted $\mathrm{Au}$ ions. However, they are know to give a very good description of the high energy interaction of such particles. They describe the initiation of the energy exchange with the surface atoms well enough to allow the formation of the ensuing collision cascade in the graphite target, which is well described by a many body potential.

The target used in this study is a graphite lattice of dimension $100 \AA \times 100 \AA$, consisting of 13 atomic layers. In the case of the Au cluster impacts

Table 1

Parameters used in the simulations

\begin{tabular}{|c|c|c|c|c|c|c|}
\hline & $\begin{array}{l}\text { Mass } \\
(\mathrm{amu})\end{array}$ & $\begin{array}{l}\text { Energy } \\
(\mathrm{keV})\end{array}$ & $\begin{array}{l}\text { Velocity } \\
(\mathrm{m} / \mathrm{s})\end{array}$ & $\begin{array}{l}\text { Momentum } \\
(\mathrm{amu} \mathrm{km} / \mathrm{s})\end{array}$ & $\begin{array}{l}\mathrm{P} / \mathrm{n}(\mathrm{amu} \mathrm{km} / \mathrm{s} / \\
\text { atom) }\end{array}$ & $\begin{array}{l}\text { Energy/n } \\
\text { (keV/atom) }\end{array}$ \\
\hline \multirow[t]{3}{*}{$\mathrm{C}_{60}$} & 720 & 1000 & 16370 & 11787 & & 17 \\
\hline & & 4000 & 32741 & 23573 & 393 & 67 \\
\hline & & 12000 & 56709 & 40830 & 681 & 200 \\
\hline \multirow[t]{3}{*}{$\mathrm{Au}_{4}$} & 788 & 1000 & 15648 & 12331 & 3083 & 250 \\
\hline & & 4000 & 31296 & 24662 & 6165 & 1000 \\
\hline & & 12000 & 54207 & 42715 & 10679 & 3000 \\
\hline \multirow[t]{3}{*}{$\mathrm{C}_{100}$} & 1200 & 2000 & 17933 & 21520 & 215 & 20 \\
\hline & & 5000 & 28354 & 34025 & 340 & 50 \\
\hline & & 20000 & 56709 & 68051 & 681 & 200 \\
\hline \multirow[t]{3}{*}{$\mathrm{Au}_{6}$} & 1182 & 1500 & 15648 & 18496 & 3083 & 250 \\
\hline & & 6000 & 31296 & 36992 & 6165 & 1000 \\
\hline & & 18000 & 54207 & 64073 & 10679 & 3000 \\
\hline
\end{tabular}


a target of 25 layers has been used. This contains approximately 50000-90000 atoms. The initial temperature of the lattice is $0 \mathrm{~K}$. Free boundaries are used in conjunction with a large lattice to avoid reflection of energy back from the sides of the simulated micro crystallite. This limits the total time that can be simulated as the energy is contained within the system and is not dissipated through out the bulk. However, the main conclusions of this work occur with in a safe time period of the simulation $(<1 \mathrm{ps})$, before much of the implanted energy has even reached the sides of the simulated volume.

\section{Results and discussion}

Simulations of single particle impacts on solid surfaces can readily be performed for amorphous targets using the TRIM code [17]. Using TRIM we can investigate the expected differences between $\mathrm{Au}$ and $\mathrm{C}$ projectiles of the same velocity. From Table 1 we wish to compare $17 \mathrm{eV}$ carbon ions with $250 \mathrm{eV}$ gold ions, $67 \mathrm{eV}$ carbon with $1 \mathrm{keV}$ gold and $200 \mathrm{eV} \mathrm{C}$ with $3 \mathrm{keV}$ gold. In Fig. 1 we summarise these TRIM simulations. First of all we should note that even in the highest energy $\mathrm{C}_{60}$ impact the individual carbon particles have slightly less kinetic energy than the individual gold parti- cles in the lowest energy $\mathrm{Au}_{4}$ cluster. From Fig. 1 it is clear that the $\mathrm{Au}$ atoms penetrate deeper into the graphite target than do the $\mathrm{C}$ atoms for the same velocity. Also the TRIM simulations show that the damage due to the $\mathrm{Au}$ particles will tend to lie between the implanted particles and the surface, whereas the damage due to the carbon particles will tend to surround the implanted species. TRIM does not detect any sputtered particles for the lowest energy impacts for either the $\mathrm{Au}$ or $\mathrm{C}$ ions. The number of displacements scales with the initial kinetic energy of the impacting particles. In a previous study of carbon cluster impacts on graphite it was shown [11] that this rule was upheld in this case also, although there was some departure from a direct scaling with single particle irradiation.

In Fig. 2 we show pictures of the cascades generated by a $\mathrm{C}_{60}$ impact and by a $\mathrm{Au}_{4}$ impact at two different times. The target atoms are coloured according to the average velocity of the particles in a $2.5 \mathrm{~A}$ radius of each site [18]. This gives an analogue of temperature on an atomistic scale. The target has been cut away so that we can "see" into the cascade. The $\mathrm{C}_{60}$ impact shows a ring propagating out from the initial impact site [18] with a dense central region at the centre. The constituents of the $\mathrm{C}_{60}$ cluster are well spread in this central core. This behaviour is typical of carbon cluster
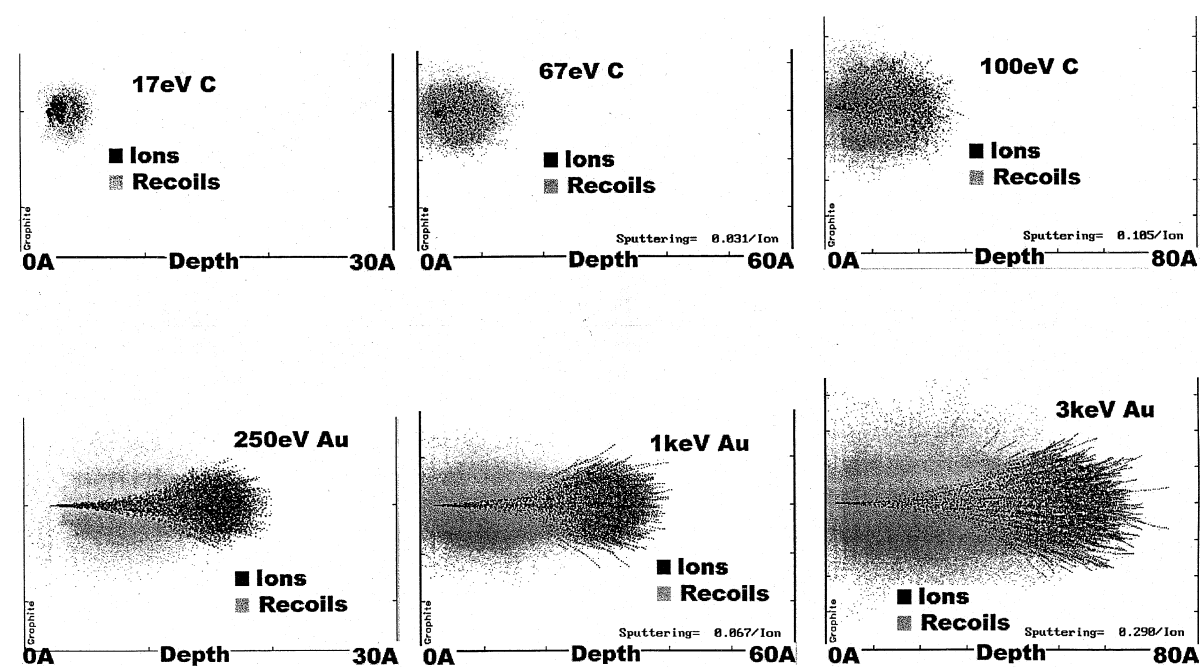

Fig. 1. TRIM simulations of 17, 67, $200 \mathrm{eV} \mathrm{C}$ ions and $250 \mathrm{eV}, 1,3 \mathrm{keV} \mathrm{Au}$ ions into amorphous carbon. 


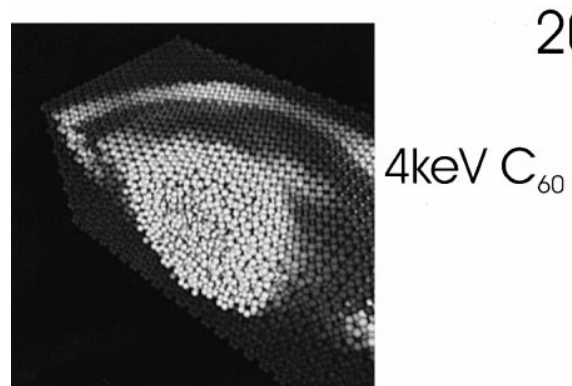

$200 f s$
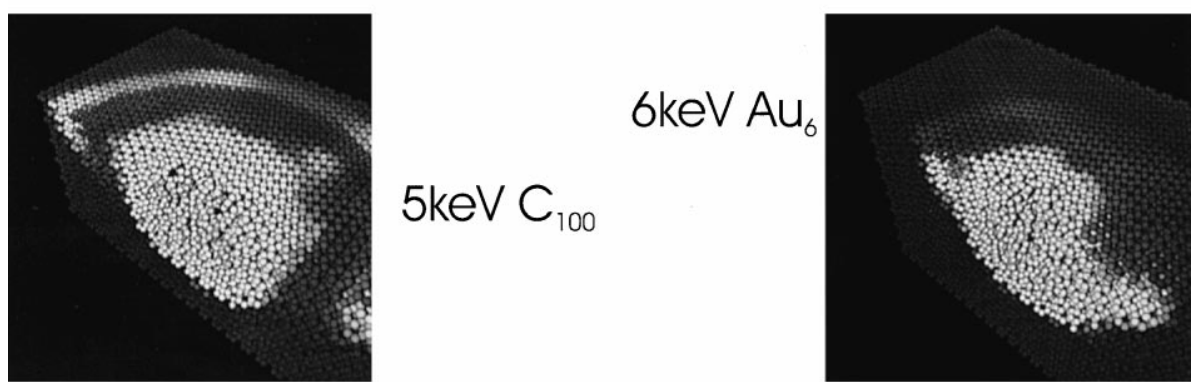

Fig. 2. Molecular Dynamics simulations of $4 \mathrm{keV} \mathrm{C}_{60}$ and $\mathrm{Au}_{4}$ cluster impacts on a graphite surface. The target has been section through the centre of the cascade to allow us to "see" inside. Colours represent the mean square velocity of the particles around each atom - see text for description.

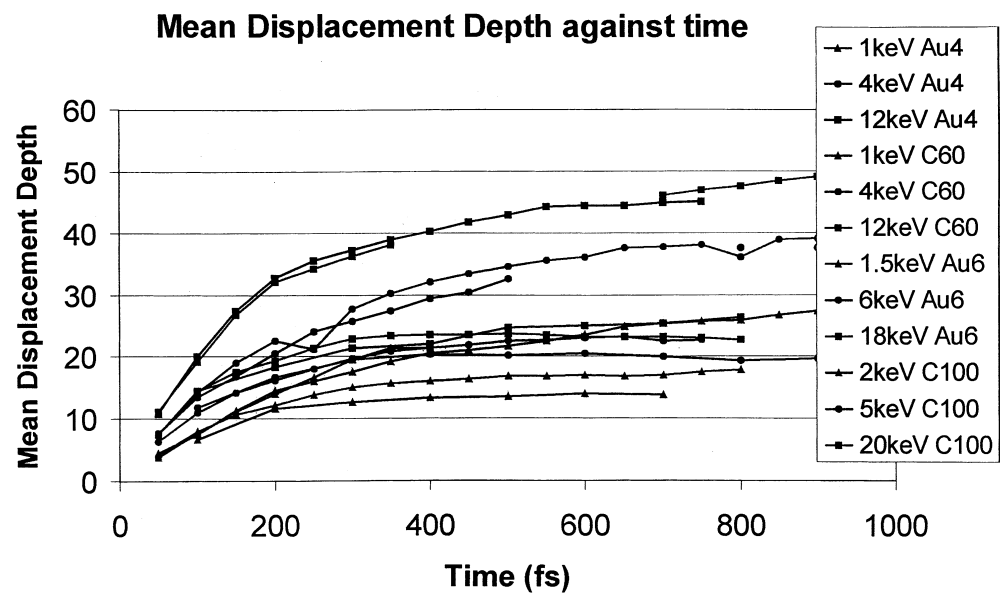

Fig. 3. The Mean Depth to which displacements of target particles are found as a function of time. These results are taken from the Molecular Dynamics calculations.

impacts at all energies studied and for different size carbon clusters from $\mathrm{C}_{2} \mathrm{H}_{4}$ to $\mathrm{C}_{300}$ [19]. The surface wave propagation from the cluster impact is very much a property of the graphite and does not appear after impact on a diamond surface [18]. $\mathrm{The} \mathrm{Au}_{4}$ cluster shows a much smaller core and the surface wave does not appear strong or to travel so far across the target. In fact, it can be seen in Fig. 2 that the cascade is less symmetrical than the $\mathrm{C}_{60}$ initiated cascade, probably due to the lower number of particles in the cluster, giving rise to discreet behaviour. The Au particles can be seen at 
the bottom of the cascade volume, not within the cascade like the $\mathrm{C}_{60}$ case. This is similar to the average behaviour demonstrated in the TRIM simulations for single particle amorphous target interactions.

One of the problems of using a graphite target in these simulations is that the layers tend to bend and vibrate about a mean position for a considerable time after the real displacement cascade has finished propagating. To avoid unnecessarily long calculation times, we show in Fig. 3 how the displacement depth changes as a function of time from the start of the calculation. The number of displacements continues to rise during this time as the bending of the layers propagates across the layers. But after about $500 \mathrm{fs}$, the depth to which the displacements propagate does not increase any further. We can be quite confident then that by taking a time of $800 \mathrm{fs}$ to measure this depth it will have stabilised.

As might be expected the penetration depths of the damage are the same for the same momentum per atom clusters. So that the $\mathrm{Au}_{4}$ and $\mathrm{Au}_{6}$ of the same velocity penetrate to the same depth and the $\mathrm{C}_{60}$ and $\mathrm{C}_{100}$ of the same velocity do likewise.
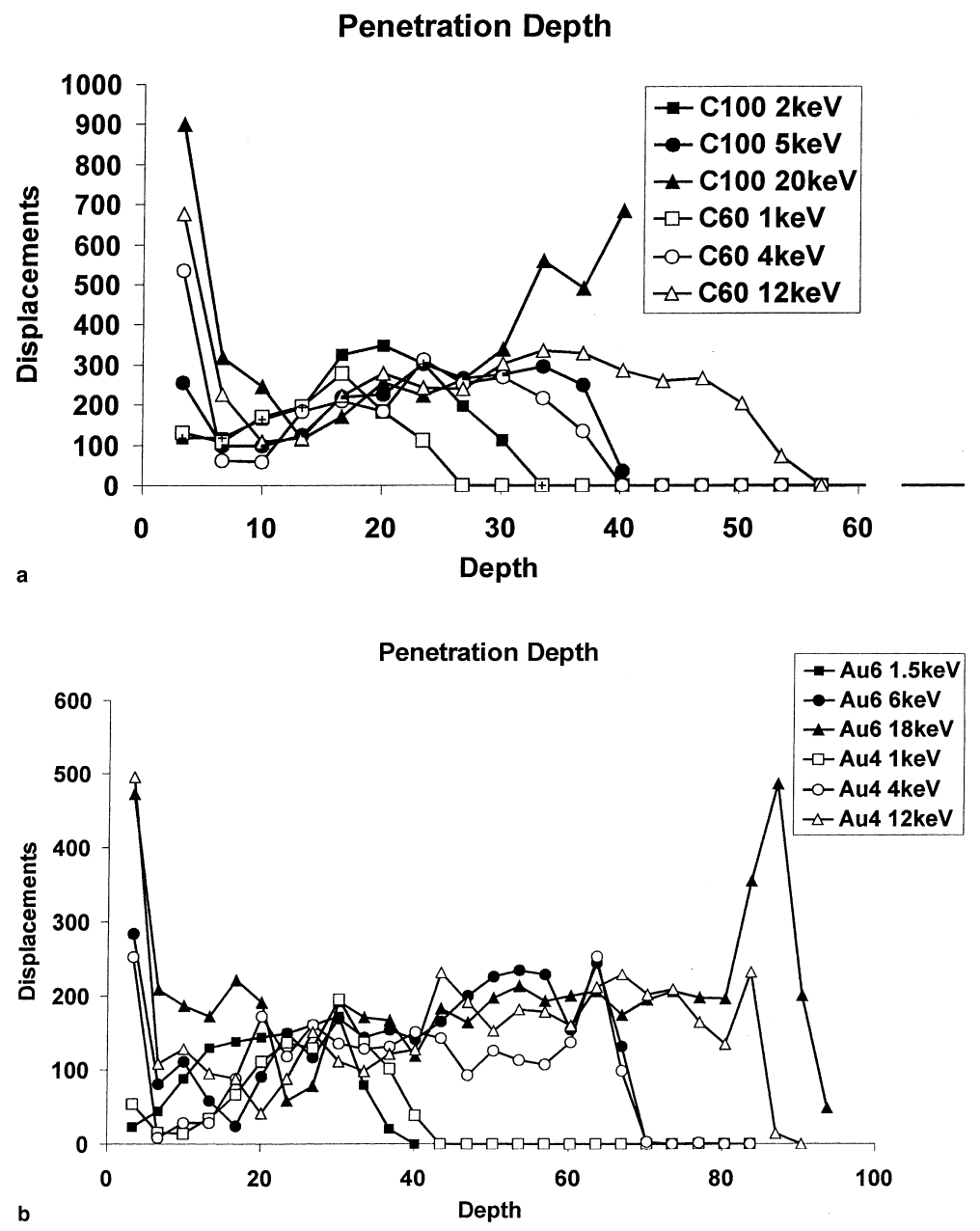

Fig. 4. Displacements versus depth for different cluster impacts on graphite from Molecular Dynamics simulation at 800 fs: (a) for carbon clusters; (b) for gold clusters. 
In Figs. 4(a) and (b) the number of "displacements" as a function of depth is plotted for the carbon clusters and gold clusters, respectively. By displacement we mean a particle which has been moved from its initial site by more than $2 \AA$. In graphite this does not necessarily mean that the particle is actually out of its lattice site. The layers can bend without breaking at least an inter-planar spacing. This makes it very difficult to determine the permanent displacement depth. But the depth to which layers are set in motion will give a useful comparison for the penetration depth. Again the behaviour of the penetration depth for different velocity particles and different mass particles is as expected. The depth of penetration is the same for clusters with similar momentum per atom. The 20 $\mathrm{keV} \mathrm{C}_{100}$ simulation was performed on a crystallite which was too thin and much material has been lost from the back surface of the target. This gives rise to the increase in displacements at the deeper layers seen in this simulation. This almost certainly would not happen if the target had been of sufficient thickness.

In Fig. 5 the number of displacements as a function of time is shown for each of the simulations. Interestingly, the number of displacements increases linearly as a function of time on the time scale up to $1.5 \mathrm{ps}$. Also this slope of these displacement versus time curves is dependent only upon the initial energy of the incoming cluster. So that in Fig. 5 there are three separate gradients, for 5,10 and $18 \mathrm{~km} / \mathrm{s}$ incident clusters. These are shown more clearly as a function of velocity in Fig. 6. These figures can be interpreted by travelling wave passing through the graphite target. Due to the nature of graphite it will propagate mainly two dimensionally, along the planes. The wave causes displacement of particles as it passes them. In previous work [18] it has been seen that similar displacement waves travel across the surface of graphite with constant velocity dependent upon the target material. If this wave propagates with constant velocity the number of displacements will be dependant upon the area of the circle of radius given by the distance travelled by the wave. If these particles all remained "displaced" then the number of displacements with time should showed a squared power dependence. Fig. 5 shows that the number of displacements increases only linearly with time. This indicates that particles are "recovering". If one considers the wave as causing displacements in a ring (i.e. that the particles inside the ring "recover") of fixed width, $\delta r$, then the number of displacements as a function of time will

Total Displacements against Time

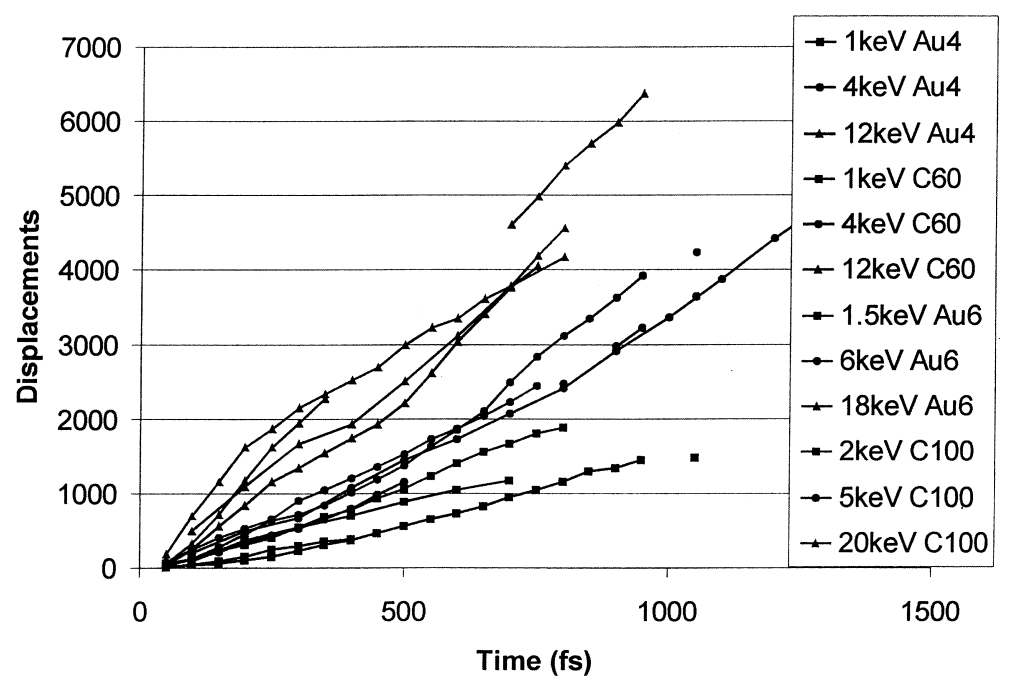

Fig. 5. Number of displaced target atoms as a function of time for all the cluster impacts on graphite. 


\section{displacement rate $\mathrm{v}$ velocity}

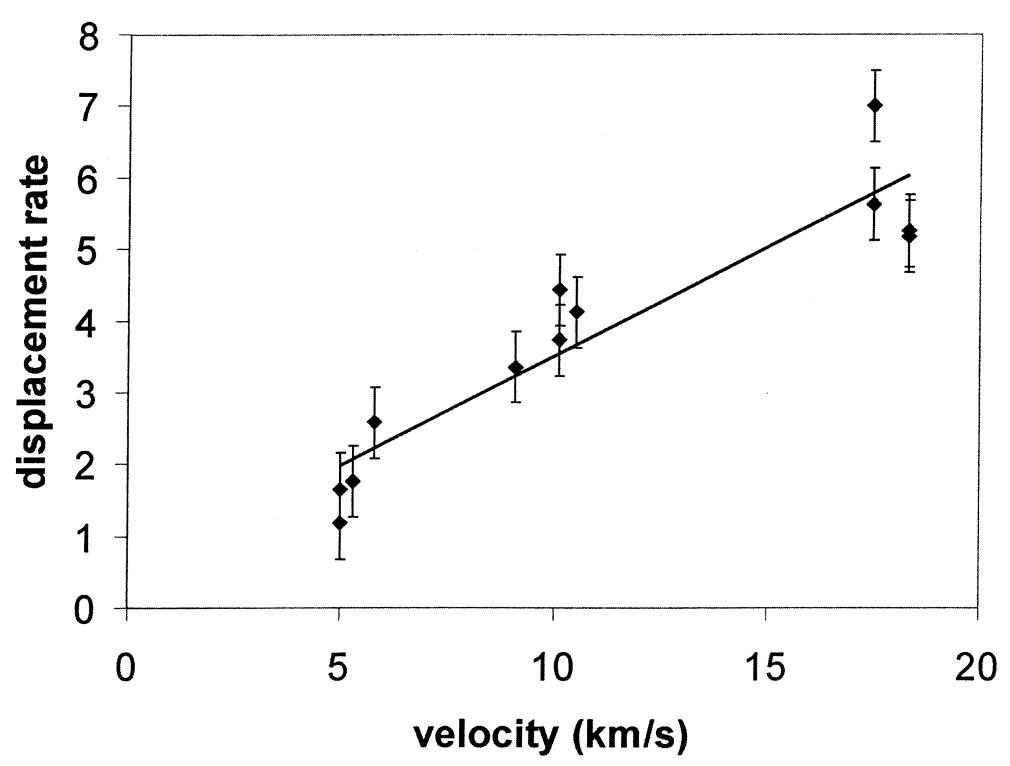

Fig. 6. The rate at which displacements are created in the target as a function of the velocity of the impacting cluster.

indeed show a linear dependence, as is observed in Fig. 5. The displacement rate shown in Fig. 6 will then be a function of this width, $\delta r$. One way of interpreting Fig. 6 then, is to say that the width, $\delta r$, of the displacement increases with increasing velocity of impact of the clusters.

\section{Conclusions}

In general then, we can conclude that the behaviour of cluster impacts is very similar to that of single particle impacts. The ratio of damage depth to implantation depth is similarly to that from single atom impacts. The formation of displaced particles is dependent upon the kinetic energy of the clusters. So that the total number of defects is the same for the same kinetic energy.

Thus it is quite easy to use clusters to tailor the defect density from each impact by adjusting the size and mass of a cluster to suit the users requirements. Further we have indications that the impact of clusters on graphite causes a displacement wave to propagate outward from the impact site, and that the magnitude of the disturbance is dependant upon the velocity of the impacting cluster.

\section{References}

[1] K. Goto, J. Makuo, D. Takiuchi, T. Sugii, I. Yamada, CP392, in: J.L. Duggan, I.L. Morgan (Eds.), Applications of Accelerators in Research and Industry, AIP Press, New York, 1997, p. 937.

[2] E.A. Schweikert, M.G. Blain, M.A. Park, E.F. Da Silveira, Nucl. Instr. and Meth. B 50 (1990) 307.

[3] M. Moseler, J. Nordiek, O. Rattunde, H. Haberland, Rad. Eff. and Defects in Solids 142 (1997) 39.

[4] G. Brauchle, S. Richard-Schneider, D. Illig, J. Rockenberger, R.D. Beck, M. Kappes, App. Phys. Lett. 67 (1995) 52.

[5] R. Webb (Ed.), Computer Simulation of Radiation Effects in Solids (COSIRES) proceedings, Radiation Effects and Defects in Solids 142 (1997).

[6] Th.J. Colla, H.M. Urbassek, Rad. Eff. and Defects in Solids 142 (1997) 439.

[7] P. Sigmund, Phys. Rev. 184 (1969) 383.

[8] R.S. Taylor, C.L. Brummel, N. Winograd, B.J. Garrison, J. Vickerman, Chem. Phys. Lett. 233 (1995) 575.

[9] R. Zaric, B. Pearson, K.D. Krantzman, B.J. Garrison, in: G. Gillen, R. Lareau, J. Bennett, F. Stevie (Eds.), 
Proceedings of the SIMS XI, Wiley, New York, 1997, p. 601.

[10] M. Benguerba, A. Brunelle, S. Della-Negra, J. Depauw, H. Joret, Y. Le Beyec, M.G. Blain, E.A. Schweikert, G. Ben Assayag, P. Sudraud, Nucl. Instr. and Meth. B 62 (1991) 8.

[11] R.P. Webb, M. Kerford, M. Kappes, G. Brauchle, Nucl. Instr. and Meth. B 122 (1997) 318.

[12] R. Smith, R.P. Webb, Proc. R. Soc. Lond. A 441 (1993) 495.

[13] D.E. Harrison Jr., Critical Rev. in Solid State Mater. Sci. 14 (1988) 1.
[14] R. Smith, D.E. Harrison Jr., B.J. Garrison, Phys. Rev. B 40 (1989) 93.

[15] D.W. Brenner, Phys. Rev. B 46 (1992) 1948.

[16] J.F. Ziegler, J.P. Biersack, U. Littmark, The Stopping and Range of Ions in Solids, Pergamon Press, New York, 1985.

[17] J.P. Biersack, L.G. Haggmark, Nucl. Instr. and Meth. 132 (1976) 647 - for original paper. We used SRIM97 available from J. Ziegler at IBM, Yorktown Heights.

[18] M. Kerford, R.P. Webb, Carbon, in press.

[19] R.P. Webb, J.J. Jimenez-Rodriguez, M. Kerford, S.R. Silva, Diamond and Related Mater. 7 (1998) 1163. 